\title{
Effect of the Combination of Trabectedin and Pegylated Liposomal Doxorubicin in a BRCA2 Mutation Carrier with Recurrent Platinum-Sensitive Ovarian Cancer
}

\author{
María Jesús Rubio Pérez \\ Servicio de Oncología, Hospital Reina Sofía de Córdoba, Córdoba, Spain
}

\section{Keywords}

Trabectedin · Ovarian cancer · Recurrent · Platinum · BRCA

\begin{abstract}
Introduction: The current standard of care for ovarian cancer is optimal cytoreduction with adjuvant chemotherapy based on a platinum/taxane combination. Although the response rate to this therapy is high, most patients ultimately relapse. Response to second-line therapy and prognosis are linked to the platinum-free interval (PFI); when both improve, the PFI increases. As a result, there is an increasing interest in the PFI extension strategies including platinum-free combinations. Case Presentation: A 50-year-old postmenopausal woman presented with ovarian serous carcinoma with peritoneal carcinomatosis. First-line neoadjuvant chemotherapy with carboplatin plus paclitaxel was initiated, followed by surgery and carboplatin plus paclitaxel chemotherapy. Eight months after the last cycle, CT revealed extensive supra- and infradiaphragmatic node involvement, and second-line chemotherapy was initiated with trabectedin and pegylated liposomal doxorubicin (PLD). Partial response was achieved and successfully maintained for 18 cycles. After the 18th cycle and a 25-month PFI, CT imaging evidenced disease progression. As the patient was a BRCA2 mutation carrier, third-line chemotherapy was initiated with carboplatin and gemcitabine every 3 weeks. After the third cycle, imaging confirmed complete response, which was maintained after the sixth
\end{abstract}


and final cycle. Maintenance treatment with olaparib was initiated. At present -6 months after the start of maintenance chemotherapy with olaparib - the patient is disease free. Conclusions: Second-line chemotherapy with a nonplatinum combination - trabectedin plus PLD - was effective in a BRCA2 mutation carrier with recurrent partially platinum-sensitive ovarian cancer.

(C) 2017 The Author(s)

Published by S. Karger AG, Basel

\section{Introduction}

Ovarian cancer is a common gynecological cancer and the fifth most frequent cause of death from cancer in women [1]. Given that this disease is asymptomatic in its early stages, late diagnosis is frequent. The current standard of care for this disease includes optimal cytoreduction with adjuvant chemotherapy following surgery and, occasionally, prior to surgery to reduce the size of the tumor. First-line chemotherapy is based on a platinum/taxane combination [2], and approximately $70-80 \%$ of patients respond satisfactorily to this combination. However, complete response is achieved in only 15\% of patients, and the majority ultimately relapse [3]. The time elapsed between the completion of first-line chemotherapy with platinum and relapse is known as the platinum-free interval (PFI). PFI helps oncologists distinguish between platinum-sensitive tumors (relapse at $\geq 6$ months after first-line chemotherapy), partially sensitive tumors (if relapse occurs 6-12 months later), and platinumresistant tumors (if relapse occurs 6 months after completion of platinum-based chemotherapy) [4].

In case of relapse, tumor response to a platinum-based regimen is directly related to the PFI. Thus, if relapse occurs in a patient with a platinum-sensitive (PFI $>12$ months) or a partially platinum-sensitive tumor (PFI $>6$ and $<12$ ), a platinum-based combination chemotherapy is recommended. Of note, a nonplatinum based combination (pegylated liposomal doxorubicin [PLD] plus trabectedin) has also been reported to be effective for platinum-sensitive tumors [5].

In 2009, trabectedin - a marine-derived antineoplastic agent initially isolated from the tunicate Ecteinascidia turbinata that is currently produced synthetically - in combination with PLD - was authorized for the treatment of patients with recurrent platinum-sensitive ovarian cancer [6]. This is currently the only nonplatinum combination approved for recurrent platinum-sensitive ovarian cancer.

Trabectedin plus PLD, as a nonplatinum combination could be especially useful for the following patient profiles: (a) patients with partially platinum-sensitive recurrent ovarian cancer, with a platinum response rate of $20-30 \%$ [6]; (b) patients who have received 2 or more previous platinum-based chemotherapy regimens, where the risk of developing a hypersensitivity reaction increases up to $27 \%$ in the 7 th cycle [7]; (c) patients who have developed a hypersensitivity reaction to platinum, which occurs in $15-20 \%$ of the patients [8]; (d) patients who reject re-treatment with platinum.

Here, we report the case of a BRCA2 mutation carrier with recurrent platinum-sensitive ovarian cancer successfully treated with second-line chemotherapy with trabectedin plus PLD followed by third-line carboplatin plus gemcitabine and maintenance therapy with olaparib. 
 Oncology}

\section{Case Presentation}

A 50-year-old postmenopausal Caucasian woman was diagnosed with high-grade ovarian serous carcinoma with peritoneal carcinomatosis in March 2013. First-line neoadjuvant chemotherapy with carboplatin and paclitaxel was administered at 3-week intervals for 4 cycles. After the fourth cycle, the patient underwent surgery, achieving optimal cytoreduction. Anatomopathological analysis revealed ovarian serous carcinoma in samples of omental implants and in 2 of 21 excised adenopathies, and high-grade papillary serous cystadenocarcinoma with frequent psammoma bodies in the ovarian samples. Surgery was followed by carboplatin plus neoadjuvant paclitaxel chemotherapy administered every 3 weeks for 4 cycles. In June 2014 - 8 months after the last cycle - PET-CT imaging showed extensive supra- and infradiaphragmatic lymph node involvement. At that point, and after an 8-month PFI, chemotherapy with trabectedin (i.v. infusion of $1.1 \mathrm{mg} / \mathrm{m}^{2}$ ) plus PLD (i.v. infusion of $30 \mathrm{mg} / \mathrm{m}^{2}$ ) every 3 weeks was initiated. Partial response was achieved. After the 12 th cycle, CA-125 levels decreased from 215 to $59(\mathrm{U} / \mathrm{mL})$, and CT imaging showed that the size of known portacaval adenopathies had decreased from 15 to $10 \mathrm{~mm}$. In November 2015, after the 18th cycle, CT imaging evidenced disease progression at supradiaphragmatic level, gallbladder and abdominal wall; CA-125 levels were elevated $(717 \mathrm{U} / \mathrm{mL})$ and ECOG performance status was 0. Given that disease progression occurred in a BRCA mutation carrier with a 25-month PFI, the management plan was to achieve platinum response and thereafter administering olaparib as maintenance therapy.

Third-line chemotherapy was initiated with carboplatin AUC4 on day 1 and gemcitabine $1,000 \mathrm{mg} / \mathrm{m}^{2}$ on days 1 and 8 every 3 weeks. After the third cycle, imaging confirmed complete response, which was maintained after the sixth and final cycle. At this point, maintenance treatment with olaparib $400 \mathrm{mg} / 12 \mathrm{~h}$ was initiated. At present (November 2016), 6 months after the start of maintenance treatment with olaparib, complete response is maintained. All treatments were well tolerated, no treatment-related toxicity was observed, and no dose reduction of any of the chemotherapy agents was necessary.

\section{Discussion}

A phase III clinical trial (the OVA-301 study, which led to the authorization of trabectedin + PLD) revealed that survival was improved with the combination of trabectedin + PLD as compared to treatment with PLD alone in patients with platinum-sensitive recurrent ovarian cancer. Notably, the difference was more significant and long-lasting in the group of patients with partially platinum-sensitive recurrent ovarian cancer (median overall survival [mOS]: trabectedin + PLD 21 months vs. PLD 17.2 months; $p=0.0056$ ) [9-11]. In a later exploratory analysis of the subset of partially platinum-sensitive patients, the subjects in the trabectedin/PLD combination arm required third-line platinum-based therapy significantly later versus those in the PLD arm. The delay in subsequent platinum treatment was largely translated into an OS extension with respect to PDL alone, suggesting that extension in the PFI may improve the response to subsequent reintroduction of platinum (mOS: trabectedin + PLD 27.7 months vs. PLD 18.7 months; $p=0.0153$ ) [12].

In vitro studies have shown that the complex mechanism of action of trabectedin which binds to the minor groove of deoxyribonucleic acid (DNA), and this triggers a cascade of events affecting DNA repair pathways - causes homologous recombination repair- 


\section{Case Reports in Oncology}

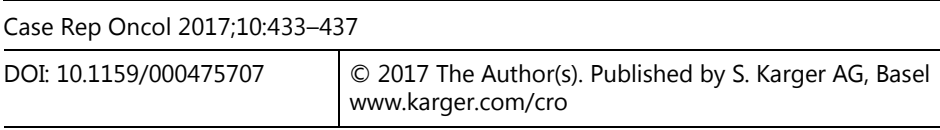
www.karger.com/cro

Rubio Pérez: Effect of the Combination of Trabectedin and Pegylated Liposomal Doxorubicin in a BRCA2 Mutation Carrier with Recurrent Platinum-Sensitive Ovarian Cancer

deficient cell lines (for example, mutations or diminished expression of BRCA1/2) to be more sensitive to trabectedin [13].

An exploratory analysis of BRCA1 mutation carriers with recurrent ovarian cancer from the OVA-301 study was conducted. The study revealed higher overall survival and progression-free survival rates in patients with recurrent platinum-sensitive ovarian cancer and mutated BRCA1 treated with trabectedin + PLD, as compared to PLD alone (median progression-free survival 13.5 vs. 5.5 months, $p=0.0002$; mOS 23.8 vs. 12.5 months, $p=0.0086$ ) [14]. Another exploratory prospective study (single-arm phase II MITO-15 trial) was performed to evaluate the efficacy of trabectedin in patients with relapsed ovarian cancer with BRCA mutation and/or BRCAness phenotype (with at least 2 previous responses to platinum). The study revealed a high response rate to trabectedin therapy in patients with platinum-sensitive tumors. However, no differences were observed in response according to BRCA status [15].

In the case reported here, second-line chemotherapy with trabectedin + PLD was effective in a BRCA2 mutation carrier with recurrent ovarian cancer partially sensitive to platinum. More specifically, partial response was reached with second-line chemotherapy with trabectedin + PLD over 18 cycles and a remarkable PFI of 25 months, with good tolerability. Subsequently third-line chemotherapy was initiated with carboplatin plus gemcitabine followed by maintenance therapy with olaparib. The patient did not present treatment-related toxicity at any time.

\section{Conclusions}

The case reported here shows that PFI was prolonged in a BRCA mutation carrier with partially platinum-sensitive recurrent ovarian cancer through the intercalation of trabectedin + PLD. It can be concluded that the trabectedin/PLD combination is an option for platinum-sensitive patients with relapsed ovarian cancer. Thus, trabectedin + PLD yields good response rates and prolongs the PFI.

\section{Statement of Ethics}

The author has no ethical conflicts to disclose.

\section{Disclosure Statement}

The author declares no conflicts of interest.

\section{References} final document of the 3rd International Gynecologic Cancer Intergroup Ovarian Cancer Consensus Conference (GCIG OCCC 2004). Ann Oncol 2005;16(suppl 8):viii7-viii12.

-3 López-Guerrero JA, Romero I, Poveda A: Trabectedin therapy as an emerging treatment strategy for recurrent platinum-sensitive ovarian cancer. Chin J Cancer 2015;34:41-49.

-4 Gabra H: Introduction to managing patients with recurrent ovarian cancer. EJC Suppl 2014;12:2-6. 
Rubio Pérez: Effect of the Combination of Trabectedin and Pegylated Liposomal Doxorubicin in a BRCA2 Mutation Carrier with Recurrent Platinum-Sensitive Ovarian Cancer

Pujade-Lauraine E: How to approach patients in relapse. Ann Oncol 2012;23(suppl 10):x128-x131. Poveda A, Ray-Coquard I, Romero I, Lopez-Guerrero JA, Colombo N: Emerging treatment strategies in recurrent platinum-sensitive ovarian cancer: focus on trabectedin. Cancer Treat Rev 2014;40:366-375.

-7 Makrilia N, Syrigou E, Kaklamanos I, Manolopoulos L, Saif MW: Hypersensitivity reactions associated with platinum antineoplastic agents: a systematic review. Met Based Drugs 2010;2010:207084.

-8 Gonzalez A: Increasing the chances for platinum-sensitive ovarian cancer patients. Future Oncol 2013;9(suppl 1):29-35.

-9 Monk BJ, Herzog TJ, Kaye S, Krasner CN, Vermorken JB, Muggia FM, et al: Trabectedin plus pegylated liposomal doxorubicin in recurrent ovarian cancer. J Clin Oncol 2010;28:3107-3114.

10 Monk BJ, Lorusso D, Italiano A, Kaye SB, Aracil M,Tanovic A, et al: Trabectedin as a chemotherapy option for patients with BRCA deficiency. Cancer Treat Rev 2016;50:175-182.

11 Mascilini F, Amadio G, Di Stefano MG, Ludovisi M, et al: Clinical utility of trabectedin for the treatment of ovarian cancer: current evidence. Oncotargets Ther 2014;7:1273-1284.

12 Poveda A, et al: Emerging treatment strategies in recurrent platinum-sensitive ovarian cancer: focus on trabectedin. Cancer Treat Rev 2014;40:366-375.

13 Lorusso D, Kahatt C, Lardelli P: Role of trabectedin in BRCA-mutated patients. Cancer Breaking News 2014;2:5-10.

14 Monk BJ, Ghatage P, Parekh T, Henitz E, Knoblauch R, et al: Effect of BRCA1 and XPG mutations on treatment response to trabectedin and pegylated liposomal doxorubicin in patients with advanced ovarian cancer: exploratory analysis of the phase 3 OVA-301 study. Ann Oncol 2015;26:914-920.

15 Lorusso D, Scambia G, Pignata S, Sorio R, Amadio G, et al: Prospective phase II trial of trabectedin in BRCA-mutated and/or BRCAness phenotype recurrent ovarian cancer patients: the MITO 15 trial. Ann Oncol 2016;27:487-493. 